\title{
Dosis de refuerzo de vacuna SARS CoV-2 para la prevención de COVID-19: Desafío en un escenario con incertezas
}

\author{
Dr. Eduardo López Mora ${ }^{1}$ \\ Dr. Jorge Espinoza Rojas ${ }^{2}$ \\ Dra. Jeannette Dabanch Peña ${ }^{3,5}$ \\ Dr. Rodrigo Cruz Choappa ${ }^{4,5}$
}

\begin{abstract}
${ }^{1}$ Residente Infectología Universidad de Valparaíso. Hospital San Camilo
${ }^{2}$ Residente Infectología Universidad de Valparaíso. Hospital Gustavo Fricke

${ }^{3}$ Hospital Clínico de la Universidad de Chile

${ }^{4}$ Hospital de Quilpué y Hospital Clínico IST

${ }^{5}$ Centro de Diagnóstico e Investigación de Enfermedades Infecciosas (CDIEI)

Universidad de Valparaíso
\end{abstract}

2 de agosto 2021

\section{INTRODUCCIÓN}

Al 16 de julio pasado existían 108 vacunas en etapa clínica de desarrollo, 28 de ellas con estudios en fase III y 6 autorizadas por la Organización Mundial de la Salud (OMS) según datos publicados al 3 de junio (AstraZeneca/Oxford, Johnson and Johnson/Janssen, Moderna, Pfizer/BionTech, Sinopharm, Sinovac) $(1,2)$. A la vez, las entidades regulatorias como la FDA (Food and Drugs Agency) y la EMA (European Medicine Agency) han autorizado para uso de emergencia las vacunas de los productores Pfizer/BioNTech, Moderna, AstraZeneca y Janssen (3) y en Chile el Instituto de Salud Pública ha autorizado 6 vacunas (Pfizer, CoronaVac, Astrazeneca, CanSino, Janssen, Sputnik).

En las últimas semanas, en el contexto de la preocupante propagación de la variante Delta (B.1.617.2) del SARS-CoV-2, la compañía Pfizer solicitó a la FDA la autorización para la administración de una dosis de refuerzo (booster) con su vacuna BNT162b2, luego de que el análisis interino de datos provenientes de la población vacunada en Israel mostrará una reducción significativa de los anticuerpos neutralizantes a los seis meses de completado el esquema primario, solicitud que generó debate en la comunidad científica. Hasta ahora los datos disponibles sobre eficacia de la vacuna BNT162b2 en escenario de circulación de variantes sugieren que continúa siendo altamente efectiva (4,5). Los estudios para definir la eventual necesidad de una dosis de refuerzo a diferentes esquemas primarios están en etapas de reclutamiento inicial en varios países.

En respuesta a la solicitud de autorización de la dosis de refuerzo realizada por Pfizer, las entidades regulatorias de Estados Unidos publicaron una declaración conjunta (FDA, $\mathrm{CDC}$ e NIH) en la que plantean - a la fecha- la falta de evidencia que pudiese sostener la recomendación de una inmunización poblacional con dosis de refuerzo, considerando además el escenario actual, en que una parte significativa de la población aún no ha recibido un esquema completo de vacunación (7). Por otra parte, las entidades regulatorias europeas (EMA, ECDC), han manifestado una posición similar, es decir, que no se cuenta con suficiente 
Dosis de refuerzo de vacuna SARS CoV-2 para la prevención de COVID-19 - López E. et al.

evidencia para recomendar por ahora la inoculación de una dosis de refuerzo (8). La OMS ha manifestado una posición en línea con la FDA y EMA, destacando a su vez el impacto que podría implicar la recomendación de dosis de refuerzo en la disponibilidad de vacunas, en una etapa de la pandemia en que se hace urgente inmunizar a la población mundial y potenciar iniciativas como COVAX, que buscan lograr un acceso a vacunas para la población de los países con menos recursos.

Aún en un escenario de incertezas sobre dosis de refuerzo, algunos países (por ejemplo, en Reino Unido) han anunciado el inicio priorizando a poblaciones más vulnerable (9).

\section{Contexto nacional}

En Chile, la campaña de vacunación iniciada el 24 de diciembre del 2020 ha utilizado diferentes plataformas de vacunas para los esquemas primarios, siendo la vacuna inactivada CoronaVac la predominante, al 15 de julio de 2021. En efecto, de las dosis de vacuna utilizadas en nuestro país, el $73 \%$ corresponde a esta última.

En el contexto de responder a la necesidad de una dosis de refuerzo para la población vacunada en el país, el Ministerio de Salud ha anunciado el desarrollo de un estudio para evaluar una eventual estrategia en este sentido (6). Un estudio de cohorte prospectiva que incluyó a cerca de diez millones de beneficiarios de FONASA, evaluó la efectividad de CoronaVac, que para la prevención de COVID-19 fue de $65,9 \%$, de $87,5 \%$ para la de hospitalización, $90,3 \%$ para ingreso a UCI y de $86,3 \%$ para muerte (10).

En tanto el Comité Asesor de Vacunas e Inmunizaciones (CAVEI), en su documento "Recomendación del CAVEI sobre la Introducción de una dosis de refuerzo al plan de vacunación COVID-19”, menciona datos preliminares no publicados que incluyen una actualización de los análisis de efectividad de CoronaVac y, adicionalmente, de la vacuna Pfizer-BioNTech, destacando mejores resultados con esta última, esencialmente en COVID-19 sintomático. Por otra parte, en la misma publicación se da cuenta de datos preliminares de otro estudio (CoronaVac03CL) sobre CoronaVac a la primera semana de julio 2021, el cual evalúa la inmunogenicidad y la seguridad de dos grupos de edad, 18-59 y $\geq 60$ años, debiendo considerar el escaso número en el segundo grupo. La seroprevalencia de AntiS1-RBD disminuyó al menos dos veces lo alcanzado a los catorce días después de la segunda dosis. El estudio de neutralización in vitro mostró reducción de la capacidad neutralizante frente a la variante P.1 linaje B.1.1.28 con el paso del tiempo, llegando a una seroconversión y niveles GMT comparables entre el día 0 y el día 180 . Contra la cepa WT (Wuhan), los niveles GMT también fueron comparables entre el día 0 y 180 (11).

\section{Estudios sobre dosis de refuerzo}

Hasta ahora, sobre la dosis de refuerzo existen escasos reportes, comunicaciones y estudios sin revisión por pares. De hecho, un estudio efectuado en 101 pacientes receptores de trasplante de órganos sólidos que analizó la presencia de anticuerpos anti SARS- CoV-2 (no hace mención sobre anticuerpos neutralizantes) mostró baja seroprevalencia de anticuerpos en aquellos con una o dos dosis, destacando un aumento significativo luego de una tercera dosis (12). Otro estudio evaluó niveles de anticuerpos neutralizantes posterior a vacunación con CoronaVac, con enfoque en la inmunogenicidad de los esquemas primarios, el cual revela que, a los seis meses de la segunda dosis, los anticuerpos neutralizantes cayeron bajo el punto de corte de seropositividad. Al administrar una tercera dosis a los 28 días o seis meses después, hubo aumento de los niveles de anticuerpos, especialmente en el refuerzo a 6 meses después de la segunda dosis (13). 
Dosis de refuerzo de vacuna SARS CoV-2 para la prevención de COVID-19 - López E. et al.

Dentro de la estrategia nacional de inmunización, y ante la consulta de una eventual introducción de una dosis de refuerzo, el CAVEI sugirió estrategias dependiendo del objetivo: transmisión o preservación del sistema sanitario. Para la primera propone vacunar con Pfizer/BioNTech, mientras que en el otro escenario sugiere privilegiar CoronaVac, considerando el número limitado de vacunas en base de plataforma ARNm (11).

Si bien hasta ahora no hay certezas sobre la duración de la protección de las vacunas COVID-19 y en el correlato sobre pérdida de efectividad, si la hay en relación con la caída de niveles de anticuerpos neutralizantes. A pesar de lo anterior, no existe aún evidencia suficiente sobre su eficacia, menos aún sobre efectividad de una dosis de refuerzo. Hoy por hoy, una dosis de refuerzo pareciera ser una estrategia probablemente necesaria, como también contar con vacunas diseñadas para neutralizar las variantes predominantes y la ansiada cobertura poblacional a nivel global.

Todavía quedan muchas preguntas por resolver en relación a una dosis de refuerzo: ¿cuál es el momento óptimo para aplicarla?, ¿con qué tipo de vacuna se debería proceder?, ¿qué población hay que priorizar?, ¿deben considerase vacunas de segunda generación? El desafío es continuar con la caracterización epidemiológica y los estudios, así, como, también, con el desarrollo de ensayos clínicos que nos permitan responder estas y otras incertezas.

\section{REFERENCIAS}

1. WHO. COVID-19 vaccine tracker and landscape. $\quad 16 \quad$ July 2021. https://www.who.int/publications/m/item/draftlandscape-of-covid-19-candidate-vaccines.

2. WHO. COVID-19 Vaccines Advice. https://www.who.int/emergencies/diseases/nove 1-coronavirus-2019/covid-19-vaccines/advice
3. EMA. COVID-19 vaccines. 18 July 2021. https://www.ema.europa.eu/en/humanregulatory/overview/public-healththreats/coronavirus-disease-covid19/treatments-vaccines/covid-19-vaccines

4. Pfizer and BioNTech Provide Update on Booster Program in Light of the Delta-Variant. https://cdn.pfizer.com/pfizercom/202107/Delta_Variant_Study_Press_Statement_Fina 1_7.8.21.pdf?IPpR1xZjlwvaUMQ9sRn2FkePcB iRPGqw

5. Liu, J, Liu, Y, Xia, H, et al. BNT162b2elicited neutralization of B.1.617 and other SARS-CoV-2 variants. Nature (2021). https://doi.org/10.1038/s41586-021-03693-y.

6. Minsal inicia estudio para determinar respuesta inmune ante una dosis de refuerzo de la vacunación contra el COVID-19. 13 de Julio 2021. https://www.minsal.cl/minsal-iniciaestudio-para-determinar-respuesta-inmuneante-una-dosis-de-refuerzo-de-la-vacunacioncontra-el-covid-19/

7. Joint CDC and FDA Statement on $\begin{array}{llll}\text { Vaccine } & \text { Boosters. July } 8021 .\end{array}$ https://www.hhs.gov/about/news/2021/07/08/jo int-cdc-and-fda-statement-vaccine-

boosters.html

8. EMA and ECDC update on COVID-19. News $14 \quad$ July 2021. https://www.ema.europa.eu/en/humanregulatory/overview/public-healththreats/coronavirus-disease-covid19/treatments-vaccines/covid-19-vaccines

9. Most vulnerable could be offered booster COVID-19 vaccines from September. https://www.gov.uk/government/news/mostvulnerable-could-be-offered-booster-covid-19vaccines-from-september 
Dosis de refuerzo de vacuna SARS CoV-2 para la prevención de COVID-19 - López E. et al.

10. Jara A, Undurraga E, González C, Paredes F, Fontecilla T, Jara G, et al. Effectiveness of an Inactivated SARS-CoV-2 Vaccine in Chile. N Engl J Med. 2021. DOI: 10.1056 / NEJMoa2107715.

11. Recomendación del CAVEI sobre la introducción de una dosis de refuerzo al Plan de Vacunación COVID-19. https://vacunas.minsal.cl/cavei/recomendacione s-cavei/

12. Kamar N, Abravanel F, Marion O, Couat C, Izopet J, del Bello A. Three Doses of an mRNA Covid- 19 Vaccine in Solid-Organ Transplant Recipients. NEJM. 2021. DOI: 10.1056 / NEJMc2108861.

13. Pan H, Wu Q, Zeng G, Yang J, Jiang D, Deng $\mathrm{X}$, et al. Immunogenicity and safety of a third dose, and immune persistence of CoronaVac vaccine in healthy adults aged 18-59 years: interim results from a double-blind, randomized, placebo-controlled phase 2 clinical trial. medRxiv. 